\title{
Mkn 463 field observed by BeppoSAX
}

\author{
R. Landi ${ }^{1,2}$ and L. Bassani ${ }^{1}$ \\ 1 ITeSRE/CNR, via Piero Gobetti 101, 40129 Bologna, Italy \\ 2 Dipartimento di Fisica, Università di Bologna, via Irnerio 46, 40129 Bologna, Italy
}

Received 1 August 2001 / Accepted 24 September 2001

\begin{abstract}
In this work we present the observation of the Mkn 463 field performed with the MECS instrument onboard BeppoSAX in the 1.8-10.5 keV band. The Mkn 463 field is an example of an extragalactic field crowded with absorbed X-ray sources: apart from the Seyfert 2 galaxy Mkn 463 and the well known QSO PG 1352+183 (the only object showing no absorption), two other objects are detected with a column density in excess of the galactic value. The first 1SAX J1353.9+1820 is a red QSO from the BeppoSAX High Energy Large Area Survey (HELLAS). The second 1SAX J1355.4+1815 is optically unidentified, but its X-ray spectral characteristics indicate that it too is an AGN hidden behind a large column density.
\end{abstract}

Key words. X-rays: galaxies - galaxies: Seyfert - galaxies: individual: Mkn 463

\section{Introduction}

Obscured active galactic nuclei (AGN) or type 2 objects, in which the power source lies behind a significant column density, are increasingly found in X-ray surveys as instruments become more and more sensitive. How common these objects are is still quite uncertain. Optical and Far-Infrared based estimates suggest a ratio of Seyfert 2 to Seyfert 1 ranging from 2 to 4 (Osterbrock \& Martel 1993; Ho et al. 1997; Schmitt 2001), but these values very likely underestimate the number of heavily absorbed objects (those with $N_{\mathrm{H}}>10^{24} \mathrm{~cm}^{-2}$, or Compton thick) which can be identified only with X-ray surveys; in particular there may be as many Compton thick Seyfert 2s as Compton thin ones (Bassani et al. 1999; Risaliti et al. 1999), implying a Seyfert 2 to Seyfert 1 ratio in the range $3-5$. This fraction can be even larger based on a simple argument recently discussed by Matt et al. (2000). The three nearest AGN (CenA, NGC 4945 and the Circinus Galaxy all within $4 \mathrm{Mpc}$ ) are all heavily obscured with column densities $\geq 10^{23} \mathrm{~cm}^{-2}$. The probability of finding a relatively unobscured AGN so close is only 0.05 , i.e. 60 times less than observed. Only if obscured AGN outnumber unobscured ones by about a factor of 10 to one does the above probability exceed $2 \%$. Here we show

Send offprint requests to: R. Landi, e-mail: landi@tesre.bo.cnr.it observationally how common absorbed sources can be. During a BeppoSAX observation of the sky region surrounding Mkn 463, a highly absorbed Seyfert 2 galaxy itself, we have found two other nearby sources which are characterized by a high column density. A fourth object detected in the same field was found to be of type 1. The probability of finding an absorbed $\mathrm{X}_{-}$ ray source in a MECS field of view of 0.8 square degrees can be estimated on the basis of the BeppoSAX HELLAS (High Energy Large Area Survey) survey Log $N-$ $\log S$ (Vignali 2000). This survey detects a higher fraction $(\sim 20 \%)$ of absorbed AGN with respect to other X-ray surveys. At the $2-10 \mathrm{keV}$ flux limit of $\sim(1-2) \times 10^{-13} \mathrm{erg} \mathrm{cm}^{-2} \mathrm{~s}^{-1}$ of our observation, we expect one HELLAS source per field of view (or 0.2 absorbed objects), i.e. at least a factor of 3 lower than observed. Again our results point to a large fraction of absorbed objects in the local universe. The true value of this fraction will eventually emerge as the sky is surveyed at progressively better sensitivity and/or higher X-ray frequencies.

\section{Observation and data reduction}

This work concerns the observation of the Seyfert 2 Mkn 463 field performed by the the Medium Energy Concentrator Spectrometer (MECS; Boella et al. 1997a) on-board BeppoSAX (Boella et al. 1997b). 
The observation was divided into three separate shots of the same field of view: the effective exposure times for the three observations are $5.3 \times 10^{3} \mathrm{~s}$ (Obs. 1, July 3rd 1998), $28.4 \times 10^{3} \mathrm{~s}$ (Obs. 2, July 5th 1998) and $53.2 \times 10^{3} \mathrm{~s}$ (Obs. 3, 28th 1998) respectively.

The target source, Mkn 463, was intentionally not onaxis, so as to also include in the MECS field of view a nearby quasar bright in X-rays, PG $1352+183$.

The two longest observations (Obs. 2 and Obs. 3) resulted in a very complex and interesting field of view; the third one was too short for any convincing evidence of sources. In order to improve the imaging quality of the data, the two longest observations were summed to produce a combined image of this sky region.

In the $1.8-10.5 \mathrm{keV}$ band (Fig. 1) two more sources were detected by the MECS in addition to Mkn 463 and PG $1352+183$.

With respect to previous observations, the most interesting result is the detection of a new $\mathrm{X}$-ray source, 1SAX J1355.4+1815. The source is located at $\alpha(2000)=$ $13^{\mathrm{h}} 55^{\mathrm{m}} 26.4^{\mathrm{s}}, \delta(2000)=+18^{\circ} 16^{\prime} 32.0^{\prime \prime}, \sim 10^{\prime}$ south of Mkn 463. The uncertainty associated with the source position is $1^{\prime}$ (90\% confidence level). This source is not identified optically either in NED or SIMBAD, although it appears as $1 \mathrm{AXG} 135526+1816$ in the $A S C A-$ GIS catalogue (Ueda et al. 2001) with a 2-10 keV flux of $2.7 \times 10^{-13} \mathrm{erg} \mathrm{cm}^{-2} \mathrm{~s}^{-1}$. At soft $\mathrm{X}$-ray energies, the object is listed as 1WGA J1355.4+1816 with a count rate ranging from $0.002-0.006$, i.e. a factor of 50 or so dimmer than PG 1352+183. Finally, a fourth source located at $\alpha(2000)=13^{\mathrm{h}} 53^{\mathrm{m}} 55.8^{\mathrm{s}}, \delta(2000)=+18^{\circ} 19^{\prime} 36.8^{\prime \prime}$ is identified with a previously discovered BeppoSAX object belonging to the HELLAS survey: 1SAX J1353.9+1820 optically identified with a broad line AGN at $z=0.217$. The $\mathrm{H} \alpha$ equivalent width $(\sim 90 \AA)$, the absence of $\mathrm{H} \beta$ and the BeppoSAX hardness ratio indicate significant reddening of the nuclear radiation. Furthermore, the presence of a broad Balmer line and the properties of the optical spectrum allow us to classify this object as a fairly lowluminosity red quasar (Vignali et al. 2000). In the following, we describe the spectral properties of all four sources detected during this observation.

\section{Spectral analysis}

For each individual observations, spectral data were extracted from regions centered on each source whose radius depends on the source intensity: we chose a radius of $4^{\prime}$ for 1SAX J1355.4+1815 and PG $1352+183$ and $2^{\prime}$ for Mkn 463 and 1SAX J1353.9+1820.

The background subtraction for the on-axis source was performed using blank sky spectra extracted from the same region of the source. Because of their faintness, for the off-axis sources we used both "blank sky" background spectra and local background spectra (extracted from a region with a radius analogous to the source extraction radius), finding no significant difference between the background subtracted spectra obtained with the two

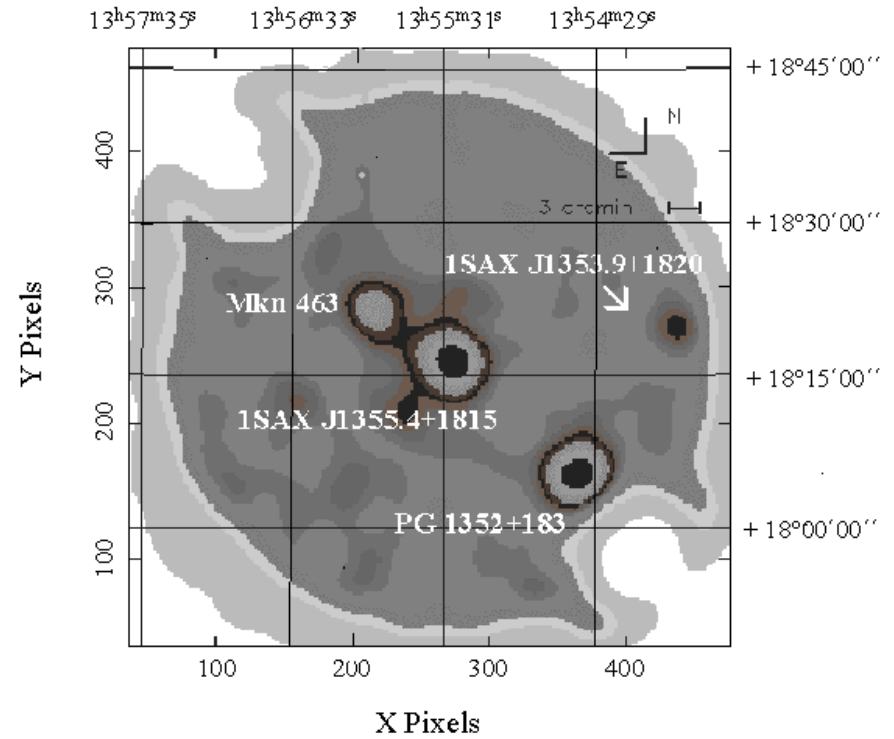

Fig. 1. MECS image of the sky region surrounding Mkn 463 obtained by summing Obs. 2 and Obs. 3 .

methods. Thus, we decided to use in our spectral analysis the local background for the off-axis sources.

The data were rebinned in order to sample the energy resolution of the detector, with an accuracy proportional to the count rate. The spectral analysis was performed with the XSPEC 10.0 package, and using the instrument response matrices released by the BeppoSAX Science Data Center in September 1997. For the off-axis sources we used the appropriate ancillary response files to avoid the effects of the vignetting due to the mirrors. All quoted errors correspond to $90 \%$ confidence intervals for one interesting parameter $\left(\Delta \chi^{2}=2.71\right)$. Unfortunately individual observations provided of too data poor quality to allow a spectral study of each source. For this reason, we tested the possibility of summing observations together for spectral analysis. However, since variability on long timescales is common in active galaxies and the measurements were taken in quite different periods, we first analyzed, for each source and for each instrument, the two longest data sets together (Obs. $2+$ Obs. 3), but allowing for a normalization constant in the simple power law fit adopted in the first instance. The results of this test listed in Table 1 indicate that the normalization factors are always compatible with 1 within errors and therefore also we combined Obs. 2 and Obs. 3 for spectral analysis.

The absorption of $\mathrm{X}$-rays due to our galaxy in the direction of each object, which amounts to $\sim 2 \times 10^{20} \mathrm{~cm}^{-2}$ (Dickey \& Lockman 1990), is fixed in all models used for the spectral analysis.

\subsection{SAX J1355.4+1815: An unidentified X-ray source}

We first fit the data with a single power law to search for any extra feature: this fit gives a slightly flat index 
Table 1. BeppoSAX MECS sources count rates and normalization factors of the two longest observations (Obs. 2 and Obs. 3 ) in the Mkn 463 field.

\begin{tabular}{l|c|c|c}
\hline & $\begin{array}{c}\text { Obs. 1 } \\
\text { Count rate }\left(\times 10^{-3} \mathrm{~s}^{-1}\right)\end{array}$ & $\begin{array}{c}\text { Obs. } 2 \\
\text { Count rate }\left(\times 10^{-3} \mathrm{~s}^{-1}\right)\end{array}$ & Normalization Factor \\
\hline Mkn 463 & $1.61 \pm 0.41$ & $2.4 \pm 0.3$ & $0.59_{-0.31}^{+0.39}$ \\
PG 1352+183 & $7.68 \pm 0.77$ & $6.0 \pm 0.6$ & $1.20_{-0.26}^{+0.30}$ \\
1SAX J1355.4+1815 & $4.85 \pm 0.65$ & $5.2 \pm 0.5$ & $0.86_{-0.23}^{+0.26}$ \\
1SAX J1353.9+1820 & $1.02 \pm 0.34$ & $0.9 \pm 0.2$ & $0.90_{-0.62}^{+0.94}$ \\
\hline
\end{tabular}

$\left(\Gamma=1.45_{-0.29}^{+0.32}\right)$ and a $\chi^{2} / \nu=11.2 / 17$. If we introduce intrinsic absorption in the source the fit is improved $\left(\chi^{2} / \nu=\right.$ $9.8 / 16$, see Fig. 2), but the spectrum is more canonical, being characterized by a $\Gamma=1.95_{-0.70}^{+0.10}$ and by an absorbing column density $N_{\mathrm{H}}=\left(1.73_{-1.73}^{+2.90}\right) \times 10^{22} \mathrm{~cm}^{-2}$. Inspection of Fig. 2 indicates that some residual emission may be present around 6-7 keV, suggesting the introduction in the fit of a narrow Gaussian emission line. The line turns out to be centered at $6.5 \pm 0.3 \mathrm{keV}$ and has an equivalent width $(E W)$ of $1.4_{-1.0}^{+0.7} \mathrm{keV}$. Fixing the line rest frame energy at $6.4 \mathrm{keV}$ provides an upper limit to the source redshift of 0.03 . The addition of the line provides an improvement in the fit $\left(\Delta \chi^{2}=5.4\right.$ for two additional parameters) which is significant at more than the $90 \%$ confidence level. If the line width is allowed to vary the best fit value is $0.24_{-0.24}^{+0.52} \mathrm{keV}$, i.e. consistent with being zero. The fit with the line gives a too steep power law $(\Gamma=2.5)$ for an AGN, although the uncertainty is large; if the power law index is fixed to 1.9 , a value more appropriate to an AGN X-ray spectrum, the column density reduces to $(1.1 \pm 1.0) \times 10^{22} \mathrm{~cm}^{-2}$ and the line $E W$ becomes $920_{-714}^{+650} \mathrm{eV}\left(\chi^{2} / \nu=7.4 / 15\right)$. The $2-10 \mathrm{keV}$ observed flux is $4.6 \times 10^{-13} \mathrm{erg} \mathrm{cm}^{-2} \mathrm{~s}^{-1}$, corresponding to an absorption-corrected luminosity $\leq 2 \times 10^{42} \mathrm{erg} \mathrm{s}^{-1}$. The observed flux suggests a variation of a factor of $\sim 2$ with respect to the $A S C A /$ GIS data. The line $E W$ is too high $(\geq 200 \mathrm{eV})$ to be produced by transmission in the observed absorbing medium, indicating the presence of either a higher column density than observed or a strong reflection component. Following Turner et al. (1997), the observed $E W$ can be produced by transmission and/or reflection in an absorbing cold medium with a column density of the order of $\sim 10^{23-24} \mathrm{~cm}^{-2}$. Therefore 1SAX J1355.4+1815 could be another example of an AGN hidden behind large amount of gas and dust.

\section{2. $M k n 463$}

Mkn 463 was the original target of the observation: this Seyfert 2 galaxy has been observed by $A S C A$, discussed by Ueno et al. (1996) and more recently reanalyzed by Levenson et al. (2001). The $A S C A$ spectrum was well described by a three components model (the thermal plus

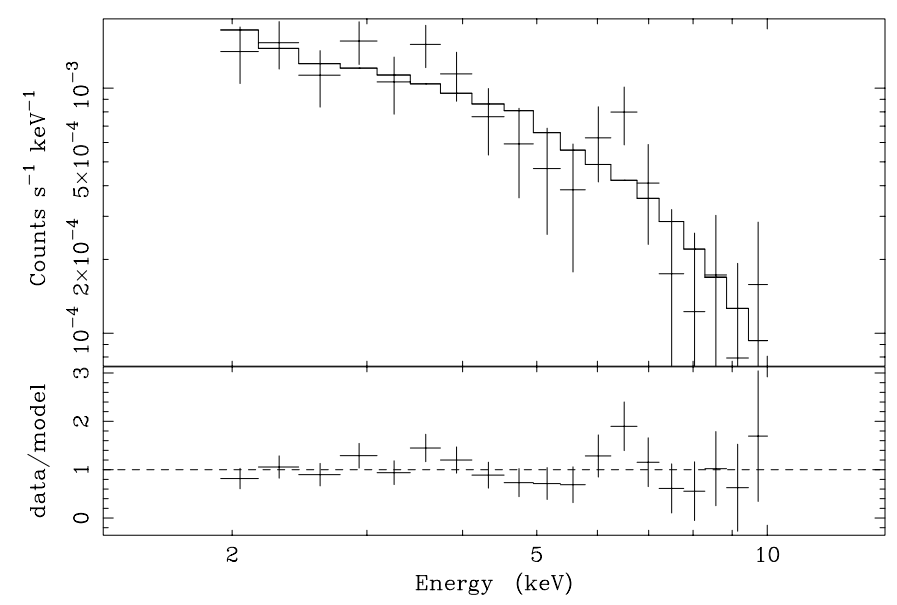

Fig. 2. Data to model ratio when MECS data of 1 SAX J1355.4+1815 are fitted with a simple power law.

scattering components which reproduce the soft $\mathrm{X}$-ray spectrum and an absorbed power law which provides the high energy emission) yielding a $2-10 \mathrm{keV}$ flux of $6.6 \times 10^{-13} \mathrm{erg} \mathrm{cm}^{-2} \mathrm{~s}^{-1}$. The measured column density was about $3 \times 10^{23} \mathrm{~cm}^{-2}$. An iron line was not evident in the data with an upper limit to the $E W$ of $890 \mathrm{eV}$. Fitting the BeppoSAX data with a simple absorbed power law provides an unrealistic inverted spectral index of -0.35 and no absorption $\left(\chi^{2} / \nu=19.5 / 16\right)$. Fixing the spectral index to a more realistic value of 1.9 requires extra absorption with respect to the galactic value of $\left(3.6_{-1.4}^{+2.1}\right) \times 10^{23} \mathrm{~cm}^{-2}\left(\chi^{2} / \nu=24.3 / 17\right)$. The fit is however not good implying a wrong modeling of the source continuum. In view of the $A S C A$ results, we introduced in the fit a scattering component with photon index equal to that of the primary emission, i.e. 1.9. In this case, we obtain an absorption of $\left(6.0_{-3.8}^{+4.1}\right) \times 10^{23} \mathrm{~cm}^{-2}$. An iron line is required/called for with a high statistical significance $\left(96 \%, \chi^{2} / \nu=12.8 / 14\right)$ : the line energy is $6.58_{-0.48}^{+0.23} \mathrm{keV}$ with an $E W=1.15_{-0.94}^{+0.77} \mathrm{keV}$, indicative of a heavily absorbed AGN. The $2-10 \mathrm{keV}$ flux was $1.1 \times 10^{-13} \mathrm{erg} \mathrm{cm}^{-2} \mathrm{~s}^{-1}$, a factor of $\sim 2$ higher than the previous $A S C A$ observation. Again the line properties as well as the ratio of the $\mathrm{X}$-ray flux compared to the [OIII] 
and Far-Infrared flux point to a heavily absorbed AGN (Bassani et al. 1999), i.e. a source with a column density $\sim 10^{24} \mathrm{~cm}^{-2}$.

\section{3. $P G 1352+183$}

PG $1352+183$ is a low redshift $(z=0.158)$ quasar previously observed by BeppoSAX on January 1998 (Mineo et al. 2001). The source spectrum is well represented by a power law with $\Gamma=2.3$ and a $2-10 \mathrm{keV}$ flux of $1 \times 10^{-12} \mathrm{erg} \mathrm{cm}^{-2} \mathrm{~s}^{-1}$. A cold iron line was also detected at a confidence level of $97 \%$; the line $E W$ was estimated to be in the range $300-1200 \mathrm{eV}$. The source is detected in each of our observations and is in fact the strongest object in the field of view. We have performed a spectral analysis on the combined data similar to that of Mineo and $\mathrm{CO}^{-}$ workers, and we obtain very similar results except for the higher flux observed: $\left(\Gamma=2.3_{-0.9}^{+1.1}, N_{\mathrm{H}} \leq 5.2 \times 10^{22} \mathrm{~cm}^{-2}\right.$ and a $2-10 \mathrm{keV}$ flux of $\left.1.8 \times 10^{-12} \mathrm{erg} \mathrm{cm}^{-2} \mathrm{~s}^{-1}\right)$. The line is also detected with a significance of $\sim 92 \%$; if the line energy is fixed at $6.4 \mathrm{keV}$, the line equivalent width is $680_{-680}^{+770} \mathrm{eV}$. The source obviously changed in flux by a factor of $\sim 2$ over a timescale of six months which is quite common in active galaxies, without any evidence of strong variation in the spectral shape. The line equivalent width is higher (although the uncertainty is large) than that usually observed in Seyfert 1 galaxies, but similar to that found in other PG quasars (Mineo et al. 2001).

\section{4. $1 S A X J 1353.9+1820$}

This source has also been observed by $A S C A$ twice and found to be characterized by an absorbed power law $(\Gamma=$ $1.2-1.3)$ with a column density of the order of $(4-6) \times$ $10^{21} \mathrm{~cm}^{-2}$. The flux remained stable between the two observations at a level of $(4-6) \times 10^{-13} \mathrm{erg} \mathrm{cm}^{-2} \mathrm{~s}^{-1}$. Despite the low statistics available, we fitted the data in order to check consistency with previous observations. We first fit the data with a simple power law model (see Fig. 3 ). The fit is satisfactory $\left(\chi^{2} / \nu=16.9 / 17\right)$ and gives a very flat spectrum characterized by a photon index $\Gamma \leq 0.87$ which is in agreement with the $A S C A$ results (Vignali et al. $2000)$. Fixing the photon index to 1.9 , the absorbing column density turned out to be of $\left(4.7_{-3.5}^{+7.0}\right) \times 10^{22} \mathrm{~cm}^{-2}$, only marginally compatible with that measured by $A S C A$.

The unabsorbed $2-10 \mathrm{keV}$ flux and luminosity are $\sim 3.6 \times 10^{-13} \mathrm{erg} \mathrm{cm}^{-2} \mathrm{~s}^{-1}$ and $4.6 \times 10^{43} \mathrm{erg} \mathrm{s}^{-1}$. The flux value is lower by a factor of two with respect to that measured with $A S C A$, while the $5-10 \mathrm{keV}$ flux is $2.4 \times 10^{-13} \mathrm{erg} \mathrm{cm}^{-2} \mathrm{~s}^{-1}$, i.e. $\sim 30 \%$ lower than the value found in the previous BeppoSAX observation (Fiore et al. 1999).

\section{Conclusions}

The sky region surrounding Mkn 463 was found to be populated with X-ray emitting objects: apart from Mkn 463 itself (the original purpose of the observation) and a

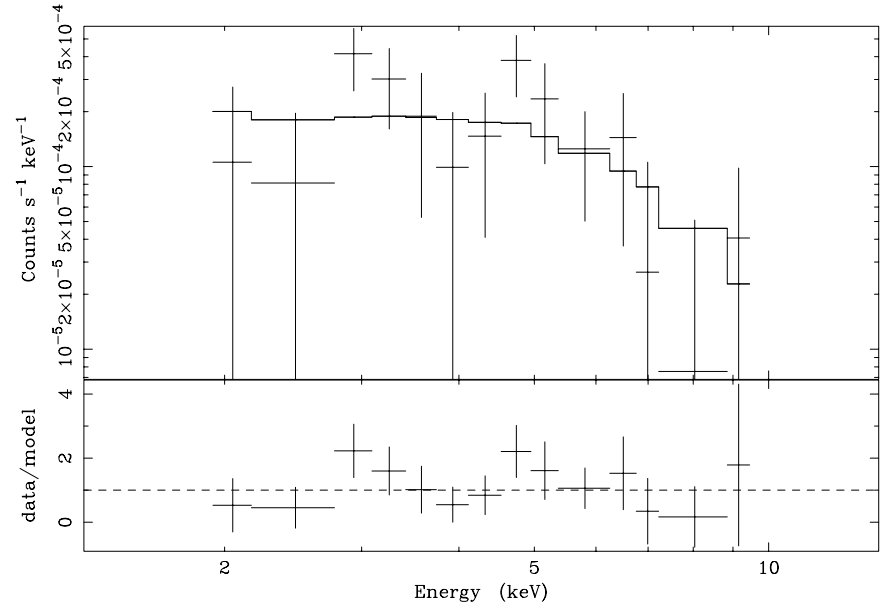

Fig. 3. Data to model ratio when MECS data of 1SAX J1353.9+1820 are fitted with a simple power law plus galactic absorption.

nearby well known QSO, we also detected two more sources. The first of the two is optically unidentified and is likely to be an absorbed object on the basis of the spectral shape and emission line characteristics, the second one is optically classified as a red QSO mildly absorbed in X-rays. The present study provides observational evidence for the high fraction of absorbed objects expected to be present among the AGN population. This result is based on simple qualitative arguments as well as on estimates of the AGN contribution to the cosmic X-ray background. Overall, the ratio of type 2 to type 1 objects is expected to exceed $3-5$; this is the ratio we observed in our observation. Our objects are, however, local and still of low luminosity in contrast to the more powerful version of the Seyfert 2 galaxy population or the long sought type 2 QSOs. The situation is now changing with Chandra and XMM which have both begun to uncover such powerful obscured AGN (Hasinger et al. 2001; Norman et al. 2001; Tozzi et al. 2001).

Acknowledgements. This research has made use of SAXDAS linearized and cleaned event files produced at the BeppoSAX Science Data Center. Financial support from the Italian Space Agency is gratefully acknowledged. We would like to thank the referee Dr. A. Orr for the very useful comments which have improved the quality of this work.

\section{References}

Bassani, L., Dadina, M., Maiolino, R., et al. 1999, ApJS, 121, 473

Boella, G., Butler, R. C., Perola, G. C., et al. 1997a, A\&AS, 122, 299

Boella, G., Chiappetti, L., Conti, G., et al. 1997b, A\&AS, 122, 327

Dickey, J. M., \& Lockman, F. J. 1990, ARA\&A, 28, 215

Fiore, F., La Franca, F., Giommi, P., et al. 1999, MNRAS, 306, L55 
Frontera, F., Costa, E., dal Fiume, D., et al. 1997, A\&AS, 122, 357

Hasinger, G., Altieri, B., Arnaud, M., et al. 2001, A\&AS, 365 L45

Ho, L. C., Filippenko, A. V., \& Sargent, W. L. W. 1997, ApJS, 112,315

Levenson, N. A., Weaver, K. A., \& Heckman, T. M. 2001, ApJS, 133, 269

Matt, G., Fabian, A. C., Guainazzi, M., et al. 2000, MNRAS, 318,173

Mineo, T., Fiore, F., Laor, A., et al. 2000, A\&A, 359, 471

Norman, C., Hasinger, G., et al. 2001, ApJ, submitted [astro-ph/0103198]

Osterbrock, D. E., \& Martel, A. 1993, ApJ, 414, 552

Parmar, A. N., Martin, D. D. E., Baudaz, M., et al. 1997, A\&AS, 122, 309
Risaliti, G., Bassani, L., Comastri, A., et al. 1999, Mem. Soc. Astron. Ital., 70, 73

Schmitt, H. R. 2001, BAAS, 198, 3601

Tozzi, P., Rosati, P., et al. 2001, ApJ, in press [astro-ph/0103014]

Turner, T. J., George, I. M., Nandra, K., et al. 1997, ApJ, 418, 164

Ueda, Y., Ishisaki, Y., Takahashi, T., et al. 2001, ApJS, 133, 1 Ueno, S., Koyama, K., Awaki, H., Hayashi, I., \& Blanco, P. R. 1996, PASJ, 48, 389

Vignali, C., Comastri, A., Stirpe, G. M., et al. 1998, A\&A, 333, 411

Vignali, C., Mignoli, M., Comastri, A., Maiolino, R., \& Fiore, F. 2000, MNRAS, 314, 11

Vignali, C. 2000, Ph.D. Thesis, University of Bologna 